\title{
Restrição fotossintética de plantas de soja sob variação de disponibilidade hídrica
}

\author{
Photosynthetic restriction of soybean plants under variation of water availability
}

\section{Geraldo Chavarria ${ }^{I^{*}}$ Miria Rosa DurigonII Vilson Antonio Klein ${ }^{I}$ Henrique Kleber}

\section{RESUMO}

$O$ objetivo do trabalho foi caracterizar o metabolismo primário, com ênfase na atividade fotossintética, de plantas de soja sob diferente disponibilidade hídrica. $O$ experimento foi conduzido em câmara de crescimento com plantas jovens de soja cv. 'BMX Apolo RR', cultivadas sem restrição hídrica até o estádio de seis folhas trifolioladas expandidas e a sétima folha aberta. Foram estudados cinco niveis de disponibilidade hídrica (-0,004, -0,006, -0,026, -0,042 e $-0,164 \mathrm{MPa}$ ) com oito vasos (repetições) por tratamento. Avaliouse a assimilação líquida de carbono $\left(A_{L}\right)$, condutância estomática (g) e transpiração (E) na sexta folha de uma planta de cada vaso. Foi determinado o potencial da água na folha, fluorescência da clorofila,conteúdo de clorofila ( $a, b$ e total), rendimento quântico e caracterizado o crescimento vegetativo. Potenciais da água no solo de -0,026 MPa comprometem a assimilação líquida de carbono de plantas de soja em função da redução da condutância estomática. Os potenciais aplicados não influenciam na quantidade de pigmentos fotossintéticos e no crescimento vegetativo em situações de breve exposição ao estresse.

Palavras-chave: Glycine max L., potencial da água, assimilação de carbono, condutância estomática, transpiração.

\section{ABSTRACT}

The aim of the research was to characterize the primary metabolism, with emphasis on photosynthetic activity, of soybean plants under different water availability conditions. The experiment was conducted in growth chamber with young plants of soybean $\mathrm{cv}$. 'BMX Apolo RR' cultivated without water restriction up to the stage of six leaves expanded and the seventh leaf unfolded. Five levels of water availability were studied $(-0.004,-0.006,-0.026,-0.042$ and $-0.164 \mathrm{MPa})$ with eight pots (replications) per treatment. It was evaluated the net carbon assimilation $\left(A_{L}\right)$, stomatal conductance $\left(g_{s}\right)$ and transpiration $(E)$ of the sixth leaf of one plant per pot. It was determined the water potential leaf, chlorophyll fluorescence, chlorophyll content ( $a, b$ and total), quantum yield and it was characterized the vegetative growth. Soil water potential of $-0.026 \mathrm{MPa}$ compromises the net carbon assimilation of soybean plants due to reduction of the stomatal resistance. The potentials applied did not influence in the amount of photosynthetic pigments andin the vegetative growth in situations of short exposition to stress.

Key words: Glycine max L., water potential, carbon assimilation, stomatal conductance, transpiration.

\section{INTRODUÇÃO}

Independentemente da região produtora do Brasil, uma das maiores limitações ao rendimento da cultura da soja (Glycine $\max$ (L.) Merr.) é a disponibilidade de água para as plantas, seja por adversidade climática (CUNHA et al., 1998), tipo ou condições de solo (DEXTER, 2004).

A demanda hídrica da cultura da soja situa-se na faixa de 550 a $800 \mathrm{~mm}$ ao longo do ciclo, sendo os momentos mais críticos os da semeadura à emergência e o de enchimento dos grãos (BERLATO et al., 1986), este último demandando uma média de 7 a $8 \mathrm{~mm}$ dia $^{-1}$ (TECNOLOGIAS DE PRODUÇÃO DE SOJA, 2008).

No processo fotossintético, a água é requerida na liberação de prótons e elétrons da etapa fotoquímica, bem como na regulação da abertura estomática, possibilitando a absorção de dióxido de carbono e a mobilização de

\footnotetext{
IFaculdade de Agronomia e Medicina Veterinária (FAMV), Universidade de Passo Fundo (UPF), Campus I, CP 611, 99001-970, Passo Fundo, RS. Brasil. E-mail: geraldochavarria@upf.br. *Autor para correspondência.
}

IIPrograma de Pós-gradução em Agronomia, FAMV, UPF, Passo Fundo, RS. Brasil. 
fotoassimilados (CHAVARRIA \& SANTOS, 2012). Nesse contexto, além da obtenção de indivíduos mais tolerantes, a restrição hídrica pelo melhoramento vegetal, torna-se fundamental o manejo adequado do solo, visando à máxima retenção e disponibilização de água às plantas, buscando alcançar altos níveis de produtividade. Nessa perspectiva, são necessários estudos buscando compreender as respostas fisiológicas das plantas em função da variação de disponibilidade hídrica no solo, no intuito de que sejam criados modelos de comportamento capazes de estimar as possibilidades dos reflexos dos manejos e da disponibilidade hídrica sobre o metabolismo das plantas. O objetivo do presente trabalho foi caracterizar o metabolismo primário, com ênfase na assimilação líquida de carbono, condutância estomática e transpiração, pigmentos fotossintéticos e crescimento vegetativo de plantas de soja, submetidas a variações de disponibilidade hídrica.

\section{MATERIAL E MÉTODOS}

O experimento foi realizadoem Passo Fundo-RS, durante os meses de janeiro a março de 2013, em câmara de crescimento climatizada, utilizando cultivar de soja 'BMX Apolo RR'. Foram semeadas, em vasos de volume de $11 \mathrm{~L}$, seis sementes por vaso e, após a germinação, foram deixadas três por vaso. Os vasos foram preenchidos com uma mistura de solo e substrato comercial (Plantmax ${ }^{\circledR}$ ) (v:v) $\left(30 \%\right.$ de argila, $10 \%$ silte, $60 \%$ de areia; $\mathrm{pH}_{\mathrm{H} 2 \mathrm{O}}$ : 6,0, P: 192,6mg dm${ }^{-3}$, K: $415 \mathrm{mg} \mathrm{dm}^{-3}$, M.O.: $>6,7 \%$, Al: $0,0 \mathrm{cmol} \mathrm{dm}^{-3}$, Ca: $7,1 \mathrm{cmol} \mathrm{dm}^{-3}, \mathrm{Mg}: 2,2 \mathrm{cmol}^{\mathrm{c}}$ $\mathrm{dm}^{-3}, \mathrm{H}+\mathrm{Al}^{\mathrm{c}}: 2 \mathrm{cmol}_{\mathrm{c}} \mathrm{dm}^{-3}, \mathrm{CTC}^{\mathrm{c}}: 12,3 \mathrm{cmol}_{\mathrm{c}} \mathrm{dm}^{-3}, \mathrm{~V}:$ $84 \%$ ), não sendo realizada adubação. As plantas se desenvolveram sem restrição hídrica até o estádio fenológico de V7 (sexta folha trifoliada expandida e a sétima folha aberta), que ocorreu 40 dias após a semeadura, quando se iniciaram as variações de disponibilidade hídrica. As plantas foram submetidas a cinco tratamentos: até que o solo atingisse potenciais mátricos de água no solo de $-0,004$ (dois dias sem água); -0,006 (quatro dias sem água); -0,026 (seis dias sem água); -0,042 (oito dias sem água); e -0,164MPa (11 dias sem água). $\mathrm{O}$ encerramento das avaliações das plantas sob déficit hídrico, e consequentemente a finalização do experimento, foi estabelecido em função do intervalo de tempo em que as plantas suportaram o estresse hídrico estipulado.

As plantas desenvolveram-se em ambiente com fotoperíodo de 10 horas de luz diária $(20,2 \mathrm{klx}$, medidos no topo da cultura), temperatura do ar de $22^{\circ} \mathrm{C}$ e umidade relativa do ar em torno de $70 \%$, controlados durante todo período do experimento.

O delineamento experimental foi efetuado em blocos casualisados com oito repetições (sendo cada vaso uma unidade experimental). Depois de realizados os tratamentos de variações de disponibilidade hídrica, foram avaliadas a assimilação líquida de carbono $\left(\mathrm{A}_{\mathrm{L}}\right)$, condutância estomática $\left(\mathrm{g}_{\mathrm{s}}\right)$ e transpiração (E) na sexta folha de uma planta de cada vaso. Tais avaliações foram realizadas uma única vez, no estádio V9 (oitava folha trifoliada expandida e a nona folha aberta), com tratamentos variando de dois a 11 dias sem água, através de um medidor de gases por infravermelho (Marca: LI-COR, Modelo: LI-6400 XT), utilizando uma curva de densidade de fluxo de fótons fotossinteticamente ativos (DFFFA) de 0, 250, 500, 1500,2000 e $2500 \mu \mathrm{mol} \mathrm{m} \mathrm{m}^{-2} \mathrm{~s}^{-1}$ através de câmara de luz (Marca: LI-COR, Modelo: LI-6400-2B), em concentração ambiente de $\mathrm{CO}_{2}$. $\mathrm{O}$ estabelecimento de uma curva de DFFFA foi necessário para definir a radiação de saturação da fotossíntese, assim como a taxa de respiração no escuro, para os distintos níveis de déficit hídrico.

Em outra planta de cada vaso, foi determinado o potencial da água da sexta folha, às 10h (máxima condutância estomática), utilizandose câmara de pressão (Marca: Soil Moisture, Modelo: 3115). Na sexta folha de uma terceira planta, ainda não utilizada nas avaliações anteriores, foi determinada a fluorescência da clorofila e o rendimento quântico do fotossistema II (Fv'/Fm'), utilizando fluorômetro (Marca: Fluorpen, Modelo: FP-100). Tais medidas foram realizadas das 10 às $12 \mathrm{~h}$, em folhas aclimatadas na presença de luz. $\mathrm{O}$ conteúdo de clorofila $a, b$ e total foi estimado através de clorofilômetro (Marca: Falker, Modelo: ClorofiLog) em trinta folhas em cada tratamento, expressos pelo índice de clorofila Falker (ICF). O ICF é um valor relativo do conteúdo de clorofila, baseado em correlações de absorbância e reflectância, e foi utilizado com o objetivo de comparar os tratamentos empregados no experimento.Todas essas avaliações foram realizadas uma única vez, no estádio V9.

A caracterização do crescimento vegetativo das plantas sobdéficit hídrico foi avaliada em uma planta de cada vaso no estádio de V9 (oitava folha trifoliada expandida e a nona folha aberta). Nesta, foi determinado o diâmetro da haste principal a $10 \mathrm{~cm}$ do nível do substrato. Foi determinada a área foliar de cada folíolo com medidor de área foliar (Marca: LI-COR, Modelo: 
3100) e obtidas as variáveis de número de folhas, área foliar unitária e área foliar total.

Amostras dosubstrato foram coletadas dos vasos,na capacidade de vaso, durante a condução do experimento, e no final do experimento (V9), para a determinação do teor de água. Ao final do experimento, amostras de solo com estrutura preservada, utilizando cilindros volumétricos $\left(100 \mathrm{~cm}^{3}\right)$, foram coletadas para determinação da capacidade de retenção de água. Essas amostras $(\mathrm{n}=10)$ foram saturadas e submetidas a tensões crescentes, utilizando funis de placa porosa e câmara de Richards, até o equilíbrio da água nas amostras. Os potenciais aplicados foram -0,001; $-0,002 ;-0,004 ;-0,006 ;-0,01 ;-0,015$ e $-0,1 \mathrm{MPa}$. Os resultados de umidade abase de volume, em função do potencial mátrico, foram ajustados ao modelo de Van Genuchten (VAN GENUCHTEN, 1980).

A partir dos dados obtidos de potencial da água na folha, taxa de $A_{L}$, taxa de $g_{s}$ e taxa de $E$, foram geradas equações de regressão. Os resultados obtidos de estimativa de pigmentos fotossintéticos, fluorescência da clorofila, rendimento quântico e crescimento vegetativo foram submetidos à análise de variância e as médias comparadas pelo teste de Tukey $(\mathrm{P}<0,05)$.

\section{RESULTADOS E DISCUSSÃO}

Os potenciais de água na folha tiveram variações entre $-0,30$ até $-0,70 \mathrm{MPa}$ (Figura 1).
Nos tratamentos com as menores disponibilidades hídricas $(-0,164 \mathrm{e}-0,042 \mathrm{MPa})$, os potenciais de água alcançados foram de $-0,53$ e $-0,70 \mathrm{MPa}$, ainda não sendo considerados pela literatura como níveis críticos de déficit hídrico. BERGONCI et al. (2000) consideraram valores de $-1,2$ a $-1,5 \mathrm{MPa}$ como críticos para a cultura do milho, sendo observado, a partir desse momento, o fechamento estomático como resposta ao déficit hídrico. Segundo RAPER \& KRAMER (1987), para a cultura da soja, valores entre $-1,0 \mathrm{MPa}$ e $-1,2 \mathrm{MPa}$ podem resultar em decréscimo das taxas fotossintéticas.

Em relação aos pigmentos fotossintéticos, não foram observadas variações nas concentrações de clorofila $a, b$ e total, nos níveis de disponibilidade hídrica fornecidos às plantas (Tabela 1). Dessa maneira, pode-se destacar que níveis de potenciais de água no solo de até $-0,164 \mathrm{MPa}$ não são suficientes para resultar em degradação de pigmentos fotossintéticos para esta cultivar; no entanto, na avaliação da fluorescência máxima da clorofila (Fm), houve diferença significativa entre os tratamentos, havendo um incremento de $23,37 \%$ na Fm, com a redução do potencial hídrico de $-0,006$ para -0,164Mpa (Tabela 1). Contudo, a fluorescência mínima (Fo) e o rendimento quântico (Qy) não apresentaram diferenças significativas (Tabela 1). A fluorescência da clorofila é considerada uma ferramenta para determinação de estresse em plantas, pois indica que a energia da radiação solar não está sendo utilizada de forma eficiente (MAXWELL \&

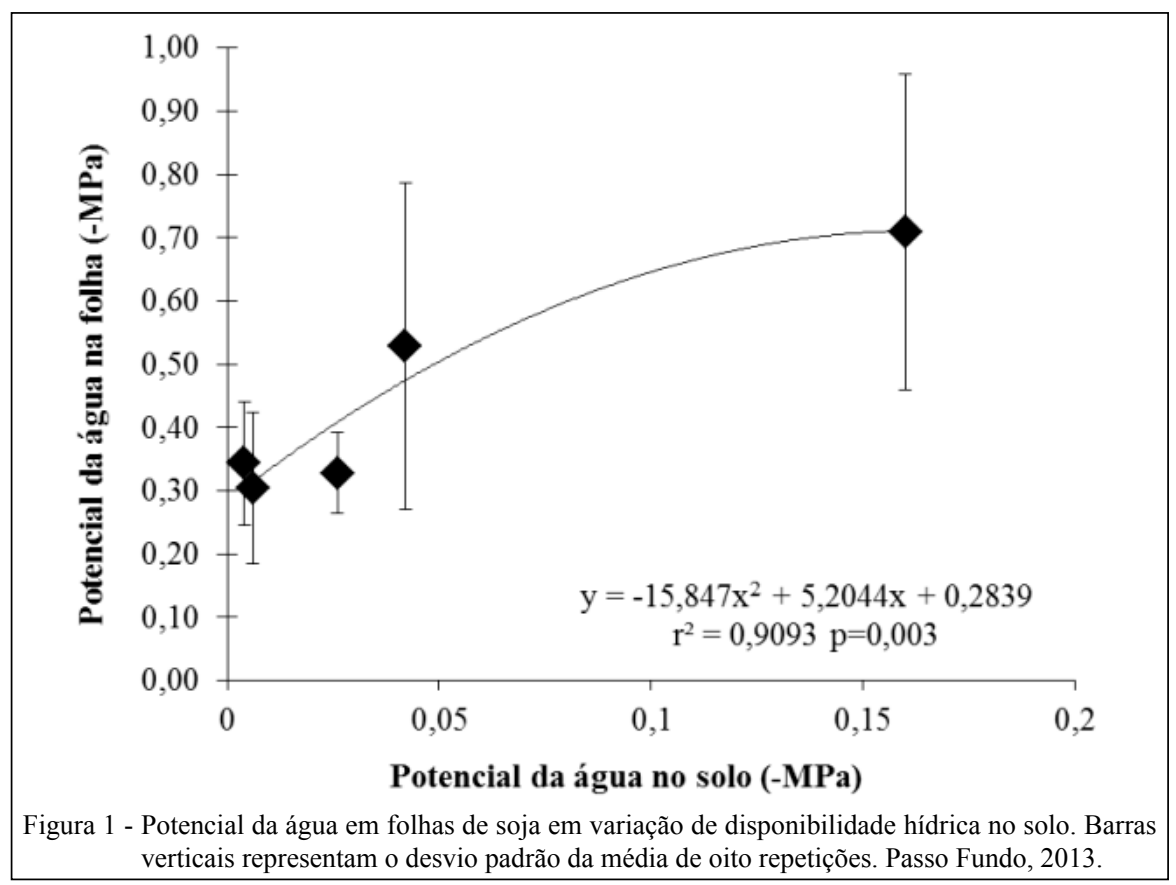

Ciência Rural, v.45, n.8, ago, 2015. 
Tabela 1 - Estimativa do conteúdo de clorofila, fluorescência inicial (Fo), máxima (Fm) e rendimento quântico (Qy) em folhas de soja com variações de disponibilidade hídrica no solo. Passo Fundo, 2013.

\begin{tabular}{|c|c|c|c|c|c|c|}
\hline Potencial de água no solo (MPa) & Clor. a & Clor. b & Clor. total & $\begin{array}{l}\text { Fluor. inicial } \\
\text { (Fo) }\end{array}$ & $\begin{array}{l}\text { Fluor. máxima } \\
\quad(\mathrm{Fm})\end{array}$ & $\begin{array}{l}\text { Rendimento } \\
\text { quântico (Qy) }\end{array}$ \\
\hline$-0,164$ & $35,22 \mathrm{~ns}^{*}$ & $12,01 \mathrm{~ns}$ & $47,23 \mathrm{~ns}$ & $378,31 \mathrm{~ns}$ & $1.499 \mathrm{a}$ & $0,75 \mathrm{~ns}$ \\
\hline$-0,042$ & 35,36 & 11,32 & 46,68 & 399,86 & $1.416 \mathrm{ab}$ & 0,71 \\
\hline$-0,026$ & 35,02 & 12,13 & 47,16 & 327,61 & $1.287 \mathrm{ab}$ & 0,75 \\
\hline$-0,006$ & 35,42 & 12,00 & 47,43 & 312,91 & $1.215 b$ & 0,74 \\
\hline$-0,004$ & 33,82 & 10,96 & 44,78 & 358,83 & $1.265 \mathrm{ab}$ & 0,72 \\
\hline $\mathrm{CV}(\%)$ & $4,55 \%$ & $12,51 \%$ & $5,94 \%$ & $20,02 \%$ & $12,73 \%$ & $5,72 \%$ \\
\hline
\end{tabular}

${ }^{*}$ Letras distintas na coluna diferem entre si com a probabilidade de $5 \%$ de erro. ns-não significativo.Clor. - clorofila;Fluor. - fluorescência da clorofila.

JOHNSON, 2000). Sendo assim, de acordo com os resultados, foi possível observar que as plantas buscaram uma dissipação de energia em função do déficit hídrico.

As maiores taxas de $\mathrm{A}_{\mathrm{L}}$ foram observadas nos potenciais de água no solo de- 0,004 e $-0,006 \mathrm{Mpa}$, com valores de 19,30 e 18,39 $\mu \mathrm{mol}$ de $\mathrm{CO}_{2} \mathrm{~m}^{-2} \mathrm{~s}^{-1}$ (Figura 2A). A partir do nível de $-0,026 \mathrm{MPa}$ de potencial de água no solo, observou-se uma redução da $A_{L}$ em 53,22\%, comparada ao maior potencial de água no solo, considerado o nível de capacidade de vaso (CASAROLI \& VAN LIER, 2008). Esse resultado pode ser explicado pelo acentuado decréscimo na $\mathrm{g}_{\mathrm{s}}$ (Figura $2 \mathrm{~B}$ ), como o observado por PEAK et al. (2004). Nos patamares de potencial de$0,164 \mathrm{e}-0,042 \mathrm{MPa}$, com maior déficit hídrico, a taxa de $A_{L}$ foi nula (Figura 2A), o que foi corroborado pela baixa taxa de E (Figuras 2C). Mesmo no tratamento com o potencial da água no solo de $-0,026 \mathrm{MPa}$ apresentando resultados de potencial da água na folha toleráveis do ponto de vista de déficit hídrico para a cultura da soja (RAPER \& KRAMER, 1987), houve redução nas taxas de $A_{L}$.

Valores nulos de $\mathrm{A}_{\mathrm{L}}$ e $\mathrm{g}_{\mathrm{s}}$ foram observados para soja, submetida à indução lenta de déficit hídrico, com conteúdo de água relativo de $60 \%$ (BERTOLLI et al., 2012). Ainda, a recuperação limitada da taxa de fotossíntese líquida em soja, submetida à indução lenta e rápida de déficit hídrico, após reidratação, provavelmente, foi causada por fatores bioquímicos (BERTOLLI et al., 2012), tais como decréscimo na síntese de RuBP (BOTA et al., 2004) e na atividade da Rubisco (PARRY et al., 2002).

Plantas sob déficit hídrico apresentam um desequilíbrio entre a captura de energia e o metabolismo, pois ocorre decréscimo na reação fotoquímica e aumento na dissipação de energia
(LAWLOR \& TEZARA, 2009). Os mesmos autores sugerem que, sob déficit hídrico moderado, ocorre decréscimo na taxa fotossintética, porém, as reações luminosas, de transporte de elétrons e de redução do $\mathrm{NADP}^{+}$são mantidas, causando um desequilíbrio energético. Isso resulta na síntese de espécies reativas de oxigênio (ROS), que danificam a enzima ATP sintase, retardando a síntese de ribulose 1,5-bifosfato (RuBP) e havendo decréscimo na atividade da rubisco.

Deve ser destacado que a taxa de $\mathrm{A}_{\mathrm{L}}$ pode ser comprometida, não somente pela redução da $\mathrm{g}_{\mathrm{s}}$, mas também pela redução da taxa de transporte de elétrons e fotofosforilação, ambos os processos da etapa fotoquímica da fotossíntese (FLEXAS et al., 2006). A deficiência hídrica, além de limitar a assimilação de carbono em diversos pontos, pode interferir no encurtamento do ciclo da soja, sobretudo no período reprodutivo, e reduzir o período de alocação de reservas para as estruturas reprodutivas (MAEHLER et al., 2003).

Em função da importância da fixação biológica de nitrogênio para a cultura da soja, é relevante destacar que esse processo pode ser comprometido pela restrição hídrica.Aproximadamente $14 \%$ do carbono assimilado vai ser repassado para as bactérias diazotróficas em troca de compostos nitrogenados (KASCHUK et al., 2010). Em caso de ocorrência de estresse hídrico, haverá redução na fixação biológica de nitrogênio e consequente redução na taxa fotossintética. Além disso, devido à maior atividade foliar e o suprimento contínuo de nitrogênio, a fixação de $\mathrm{N}_{2}$ potencialmente retarda a senescência foliar, aumentando o período de enchimento de grãos na soja (KASCHUK et al., 2010). 


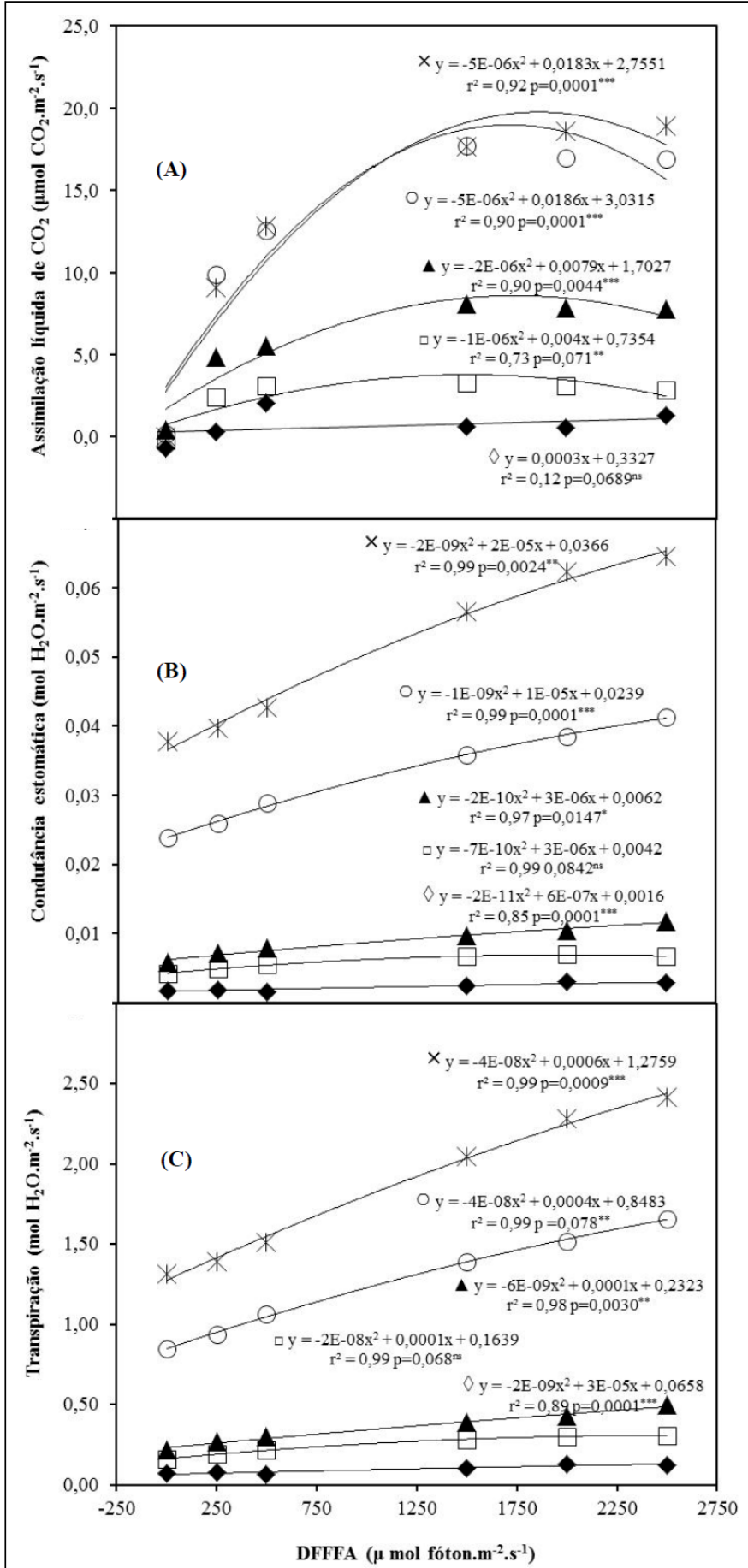

Figura 2 - Assimilação líquida de $\mathrm{CO}_{2}$ (A), condutância estomática (B) e transpiração (C) em função da densidade de fluxo de fótons fotossinteticamente ativos (DFFFA) em folhas de soja, cv. 'BMX Apolo RR', submetidas a variações de disponibilidade hídrica $(\times=-0,004 \mathrm{MPa}, \circ=-0,006 \mathrm{MPa}, \boldsymbol{\Delta}=-0,026 \mathrm{MPa}, \square=$ $-0,042 \mathrm{MPa}$ e $=-0,164 \mathrm{MPa})$. Passo Fundo, 2013.

Houve ajuste de curvas quadráticas para a taxa de $\mathrm{A}_{\mathrm{L}}$ de $\mathrm{CO}_{2}$ em função da DFFFA para os níveis de déficit hídrico de $-0,004$ a -0,042MPa e a uma curva linear para o maior déficit hídrico (-0,164MPa). A adequação de uma curva exponencial ou hiperbólica dos dados da taxa de $\mathrm{A}_{\mathrm{L}}$ de $\mathrm{CO}_{2} \mathrm{em}$ 
função da DFFFA tem sido verificada por alguns autores (PRADO \& MORAES, 1997; GOMES et al., 2006), que avaliaram a taxa de $\mathrm{A}_{\mathrm{L}}$ de $\mathrm{CO}_{2}$ sob DFFFA de até $1600 \mu \mathrm{mol}$ fótons $\mathrm{m}^{-2} \mathrm{~s}^{-1}$. No presente estudo, foi avaliada a taxa de $\mathrm{A}_{\mathrm{L}}$ de $\mathrm{CO}_{2}$ sob DFFFA de até $2500 \mu \mathrm{mol}$ fótons $\mathrm{m}^{-2} \mathrm{~s}^{-1}$, sendo observados valores máximos em aproximadamente $1500 \mu \mathrm{mol}$ fótons $\mathrm{m}^{-2} \mathrm{~s}^{-1}$ e posteriormente um decréscimo em seus valores, o que justifica a utilização de uma curva quadrática.

De acordo com as variáveis analisadas no trabalho, mesmo onde o potencial da água na folha (Figura 1) e a fluorescência da clorofila (Tabela 1) não apontaram uma restrição hídrica nas plantas de soja, foi possível observar uma restrição metabólica, representada pela diminuição da taxa de $\mathrm{A}_{\mathrm{L}}$ (Figura 2A). A diminuição na taxa fotossintética pode estar relacionada a fatores difusivos (estomáticos) ou metabólicos (não-estomáticos). O principal efeito da baixa disponibilidade de água na planta é a menor assimilação de carbono pela folha, devido ao fechamento estomático, que pode ocorrer após um déficit hídrico moderado (CHAVES \& OLIVEIRA, 2004). Já as restrições metabólicas, tais como reflexos sobre a atividade da rubisco, dosfotossistemas e o conteúdo de RuBP não são afetados até que haja um déficit hídrico severo (BOTA et al., 2004; LAWLOR \& TEZARA, 2009).

O crescimento vegetativo da soja (diâmetro da haste principal e área foliar) em função das variações de disponibilidade hídrica não foi influenciado (Tabela 2). O maior nível de déficit hídrico foi propiciado à planta por um período de 11 dias sem água, alcançando potencial de $-0,164 \mathrm{MPa}$. Em função dos resultados obtidos, esse estresse, que culminou em uma redução na taxa de $A_{L}$ em $90 \%$, não foi suficiente para refletir no crescimento em situação de breve exposição. Em situações em que o déficit hídrico seja mais prolongado, pode afetar não só fotossíntese, como foi observado, mas também a altura e o índicede área foliar. Além disso, quando o déficit hídrico desenvolve-se no campo, de forma mais lenta, pode prejudicar o crescimento vegetativo da soja, já que, em condições de vaso, ocorre limitação ao crescimento das raízes.

Esses resultados corroboram a importância do manejo de solo com vistas à melhoria das características da física do solo, pois, em última análise, quanto maior a capacidade das plantas emmovimentar água do solo para a atmosfera, maior a assimilação de carbono. De acordo com BEUTLER \&CENTURION (2003), no que concerne a plantas de soja, em latossolos, a produtividade de grãos é reduzida em situações em que o solo apresenta níveis de resistência à penetração de $2 \mathrm{MPa}$ e retenção de água a $-0,01 \mathrm{MPa}$.

\section{CONCLUSÃO}

Potenciais da água no solo iguais ou inferiores a $-0,026 \mathrm{MPa}$ provocam redução significativa da atividade fotossintética de plantas de soja, cultivar 'BMX Apolo RR', pela menor condutância estomática.

Os potenciais aplicados não influenciam na quantidade de pigmentos fotossintéticos e no crescimento vegetativo das plantas de soja no período de déficit hídrico estudado.

Tabela 2 - Desenvolvimento vegetativo de plantas de soja submetidas a variações na disponibilidade hídrica em um intervalo de tempo de 11 dias. Passo Fundo, 2013.

\begin{tabular}{llll}
\hline Potencial de água no solo (MPa) & Área foliar unitária $\left(\mathrm{cm}^{2}\right)$ & Área foliar total $\left(\mathrm{cm}^{2}\right)$ & $\begin{array}{c}\text { Número de folhas (un.) } \\
\text { Diâmetro da haste } \\
\text { principal }(\mathrm{cm})\end{array}$ \\
\hline$-0,164$ & $14,79 \mathrm{~ns}^{*}$ & $522,20 \mathrm{~ns}$ & $35,33 \mathrm{~ns}$ \\
$-0,042$ & 13,13 & 571,67 & 38,00 \\
$-0,026$ & 15,27 & 550,90 & 36,00 \\
$-0,006$ & 12,37 & 381,65 & 31,00 \\
$-0,004$ & 11,95 & 461,70 & 38,67 \\
CV $(\%)$ & $18,17 \%$ & $14,37 \%$ & $8,36 \%$ \\
\hline
\end{tabular}

*Letras distintas na coluna diferem entre si com a probabilidade de $5 \%$ de erro. ns-não significativo. 


\section{REFERÊNCIAS}

BERGONCI, J.I. et al. Potencial da água na folha como um indicador de déficit hídrico em milho. Pesquisa Agropecuária Brasileira, v.35, n.8, p.1531-1540, 2000. Disponível em: <http://www.scielo.br/scielo.php?pid=S0100204X2000000800005\&script=sci_arttext $>$. Acesso em: 15 mar. 2013. doi: 10.1590/S0100-204X2000000800005.

BERLATO, M.A. et al. Evapotranspiração máxima da soja, relações com a evapotranspiração calculada pela equação de Penman, evaporação de tanque "classe A" e radiação solar global. Agronomia Sulriograndense, v.22, n.2, p.243-259, 1986.

BERTOLLI, S.C. et al. Photosynthetic limitations caused by different rates of water-deficit induction in Glycinemax and Vignaunguiculata. Photosynthetica, v.50, p.329336, 2012. Disponível em: <http://link.springer.com article/10.1007\%2Fs11099-012-0036-4\#page-1>. Acesso em: 04 ago. 2013. doi: 10.1007/s11099-012-0036-4.

BEUTLER, A.N.; CENTURION, J.F. Efeito do conteúdo de água e da compactação do solo na produção de soja. Pesquisa Agropecuária Brasileira, v.38, n.7, p.849-856, 2003. Disponível em: <http://www.scielo.br/pdf/pab/v38n7/18207.pdf>. Acesso em: 25 fev. 2015. doi: 10.1590/S0100-204X2003000700009.

BOTA, J.et al. Is photosynthesis limited by decreased Rubisco activity and RuBP content under progressive water stress? New Phytologist, v.162, p.671-681, 2004. Disponível em: <http:// onlinelibrary.wiley.com/doi/10.1111/j.1469-8137.2004.01056.x/ pdf>. Acesso em: 04 ago. 2013. doi: 10.1111/j.14698137.2004.01056.x.

CASAROLI, D.; VAN LIER, Q.J. Critérios para determinação da capacidade de vaso. Revista Brasileira de Ciência do Solo v.32, p.59-66, 2008. Disponível em: <http://www.scielo.br/pdf/ rbcs/v32n1/07.pdf>. Acesso em: 15 maio 2013.

CHAVARRIA, G.; SANTOS, H.P. Plant water relations: absorption, transport and control mechanisms. In: MONTANARO, G.; DICHIO, B. (Org.). Advances in selected plant physiology aspects. Rijeka: Intech, 2012. v.1, p.105-132.

CHAVES, M.M.; OLIVEIRA, M.M. Mechanisms underlying plant resilience to water déficits: prospects for water-saving agriculture. Journal of Experimental Botany, v.55, n.407, p.2365-2384, 2004. Disponível em: <http://jxb.oxfordjournals. org/content/55/407/2365.long >. Acesso em: 13 set. 2013. doi: $10.1093 / \mathrm{j} \times \mathrm{b} / \mathrm{erh} 269$.

CUNHA, G.R. et al. Perda de rendimento potencial em soja no Rio Grande do Sul por deficiência hídrica. Revista Brasileira de Agrometeorologia, v.6, n.1, p.111-119, 1998.

DEXTER, A.R. Soil physical quality. Part I. Theory, effects of soil texture, density, and organic matter, and effects on root growth. Geoderma, v.120, n.3, p.201-214, 2004.
FLEXAS, J. et al. Photosynthesis limitations during water stress acclimation and recovery in the drought-adapted Vitis hybrid Richter-110 ( $\boldsymbol{V}$. Berlandierix, V.rupestris). Journal of Experimental Botany, v.60, n.8, p.2361-2377, 2009.

GOMES, F.P. et al. Photosynthetic irradiance-response in leaves of dwarf coconut palm (Cocosnucifera L. 'nana', Arecaceae): comparison of three models. Scientia Horticulturae, v.109, n.1, p.101-105, 2006. Disponível em: <http://www.sciencedirect. com/science/article/pii/S0304423806001142>. Acesso em: 29 nov. 2013. doi: 10.1016/j.scienta.2006.02.030.

KASCHUK,G.etal.Differences in photosynthetic behaviorandleaf senescence of soybean (Glycinemax [L.] Merrill) dependent on $\mathrm{N}_{2}$ fixation or nitrate supply. Plant Biology, v.12, n.1, p.60-69, 2010.

LAWLOR, D.W.; TEZARA, W. Causes of decreased photosynthetic rate and metabolic capacity in water-deficient leaf cells: a critical evaluation of mechanisms and integration of processes. Annals of Botany, v.103, p.561-579, 2009.

MAEHLER, A.R. et al. Potencial de rendimento da soja durante a ontogenia em razão da irrigação e arranjo de plantas. Pesquisa Agropecuária Brasileira, v.38, n.2, p.225-231, 2003.

MAXWELL, K.; JOHNSON, G.N. Chlorophyll fluorescence - a practical guide. Journal of Experimental Botany, v.51, n.1, p.659-668, 2000.

PARRY, M.A. et al. Rubisco activity: effects of drought stress. Annals of Botany, v.89, p.833-839, 2002.

PEAK, D. et al. Evidence for complex, collective dynamics and emergent, distributed computation in plants. Proceedings of the National Academy of Sciences of the United States of America, v.101, n.4, p.918922, 2004. Disponível em: <http:// www.pnas.org/content/101/4/918.full>. Acesso em: 20 mar. 2013. doi: $10.1073 /$ pnas. 0307811100

PRADO, C.H.B.A.; MORAES, J.A.P.V. Photosynthetic capacity and specific leaf mass in twenty woody species of Cerrado vegetation under field conditions. Photosynthetica, v.33, n.1, p.103-112, 1997. Disponível em: <http://link.springer.com/art icle/10.1023\%2FA\%3A1022183423630>. Acesso em: 29 nov. 2013. doi: 10.1023/A:1022183423630.

RAPER, C.D.; KRAMER, P.J. Stress physiology. In: WILCOX, J.R. (Ed.). Soybeans: improvement, production, and uses. Madison: American Society of Agronomy, 1987. p.589-641.

TECNOLOGIAS DE PRODUÇÃO DE SOJA - Região central do Brasil 2009 e 2010. Londrina: Embrapa Soja, 2008.262p. (Sistemas de produção, 13).

VAN GENUCHTEN, M.T.H. A closed-form equation for predicting the hydraulic conductivity of unsaturated soils. Soil Science Society of America Journal, v.44, n.1, p.892-897, 1980. 